\title{
The Vascular Flora of the Oklahoma Centennial Botanical Garden site Osage County, Oklahoma
}

\author{
Bruce W. Hoagland \\ Oklahoma Biological Survey \\ Department of Geography \\ University of Oklahoma \\ Norman, OK 73019-0575 \\ * e-mail: bhoagland@ou.edu
}

\author{
Amy Buthod \\ Oklahoma Biological Survey \\ University of Oklahoma \\ Norman, OK 73019-0575
}

This paper is a report on the results of an inventory of the vascular plants at the future site of the Oklahoma Centennial Botanical Garden in Osage County, Oklahoma. We collected a total of 293 taxa in 208 genera and 68 families. The families Poaceae and Asteraceae had the greatest number of species with 50 and 44 species respectively. Fortyone species of woody plants were present. Forty-four non-native species were present, representing $15 \%$ of the flora. No species tracked by the Oklahoma Natural Heritage Inventory were present.

\section{INTRODUCTION}

The objective of this study was to complete a floristic inventory at the future site of the Oklahoma Centennial Botanical Garden (OCBG) in southeast Osage County $\left(36.2017^{\circ} \mathrm{N}\right.$ to $36.2109^{\circ} \mathrm{N}$ and $96.0555^{\circ} \mathrm{W}$ and $\left.96.0678^{\circ} \mathrm{W}\right)$. Construction of the OCBG is scheduled to begin in late 2007 on 87 hectares (215 acres). The master plan, developed by Marshall Tyler Rausch of Pittsburgh, Pennsylvania, includes a Mexican Garden, Oklahoma Wildflower Garden, Cross Timbers Prairie and Woodland, Folk Garden, Horticultural Therapy Garden, Children's Garden, Demonstration Gardens, and others. In addition, a 17-acre lake, an amphitheater, a visitor center, education buildings, and a conservatory will be constructed (Oklahoma Centennial Botanical Garden 2007).

The OCBG site is located in the Claremore Cuesta plains geomorphic province of southeastern Osage County (Curtis and Ham 1979). Surface geology is predominantly Pennsylvanian sandstone and shale (Branson and Johnson 1979). Soils belong to the Niotaze-Darnell Association, described as moderately deep and shallow, gently sloping to steep, loamy soils over shale and sandstone (Bourlier et al. 1979). The climate is Subtropical Humid (Cf) (Trewartha 1968). Summers are warm and humid. Mean July temperature is $27.5^{\circ} \mathrm{C}$ $\left(81.5^{\circ} \mathrm{F}\right)$. Winters are relatively short and mild with a mean January temperature of $1.5^{\circ} \mathrm{C}\left(34.7^{\circ} \mathrm{F}\right)$. Mean annual precipitation is $111.7 \mathrm{~cm}$ (43.8 in) (Oklahoma Climatological Survey, 2007). Elevation ranges from 259 to $302 \mathrm{~m}$ (849.5 to 990.6 $\mathrm{ft})$. Potential natural vegetation at OCBG is post oak-blackjack forest and tallgrass prairie (Duck and Fletcher 1943). Historical land use of the site has included livestock grazing and oil exploration.

\section{METHODS}

Three collection sites were visited monthly for floristic sampling. The predominant vegetation association at these sites was classified according to Hoagland (2000). Additional collections were also made opportunistically throughout the OCBG. Collecting began in July of 2006 and continued through July of 2007. Vouchers for non-native species were made from naturalized populations only, thus 
Oklahoma Native Plant Record

Volume 7, Number 1, December 2007

excluding cultivated and ornamental plants. Specimens were processed following standard procedures and deposited at the Robert Bebb Herbarium at the University of Oklahoma (OKL). Manuals used for specimen identification included Waterfall (1973) and Steyermark (1963). Origin, either native or introduced to North America, was determined using the Plants Database (USDA-NRCS, 2007). Nomenclature follows the United States Department of Agriculture-Natural Resources Conservation Service (USDA-NRCS 2007).

\section{RESULTS AND DISCUSSION}

A total of 293 taxa of vascular plants in 68 families and 208 genera were collected at the OCBG (Appendix). Of the angiosperms, 92 species were Liliopsida and 199 were Magnoliopsida (Table). There was one species of Pteridophyta and one of Coniferophyta. Forty-one species were trees, shrubs, and woody vines. The Poaceae with 50 taxa, and the Asteraceae with 44 taxa, were the largest families. The genera Symphyotrichum (formerly Aster) and Cyperus had the most species, with seven and six species respectively. One hundred and seven taxa were annuals, 2 were biennials and 184 were perennials.

Forty-four species (15\% of the flora) in 25 families were non-native to Oklahoma. The percentage of non-native species at the OCBG is high when compared to other floristic surveys from Oklahoma, which range from 6.6\%-15\% (Hoagland and Buthod 2004; Hoagland and Johnson 2005). The greatest numbers of non-native species occurred in the Poaceae, with eleven and Fabaceae, with eight. No species tracked by the Oklahoma Natural Heritage Inventory (2007) were encountered.

Collection sites selected at OCBG occurred within four vegetation associations. A description of each vegetation category follows:
1. Quercus stellata-Quercus marilandica forest association [QSQM]

This vegetation association occupied a small percentage of the OCBG. Common associated species included Amelanchier arborea, Antennaria plantaginifolia, Baptisia bracteata var. leucophaea, Danthonia spicata, Helianthus birsutus, Hypericum bypericoides, Symphyotrichum patens, Myosotis verna, Opuntia bumifusa, Sideroxylon lanuginosum, Smilax rotundifolia, Ulmus alata, and Viburnum rufidulum.

\section{Schizachyrium scoparium-Sorghastrum nutans} [SSN]

This herbaceous grassland vegetation association occupied the greatest area at the OCBG. Soils were typically shallow with exposed cobble. Associated species included Amorpha canescens, Arnoglossum plantagineum, Callirhoe alcaeoides, Coreopsis grandiflora, Cyperus echinatus, Echinacea atrorubens, Krameria lanceolata, Lespedeza cuneata, Minuartia drummondii, and Pediomelum linearifolium.

3. Wetland and aquatic vegetation [WETL]

Wetland vegetation was restricted to a small stream bisecting the site and its associated beaver pond. Common associates included Alisma subcordatum, Ammannia auriculata, Callitriche heterophylla, Cephalanthus occidentalis, Eclipta prostrata, Fimbristylis autumnalis, Juncus brachycarpus, Ludwigia palustris, Nelumbo lutea, Polygonum pensylvanicum, Sagittaria ambigua, and Samolus ebracteatus.

4. Disturbed areas and old-field vegetation [DAOF]

Disturbed areas coincided with roadways and oil extraction sites. Common associated species included Achillea millefolium, Aegilops cylindrica, Capsella bursapastoris, Carduus nutans, Convolvulus arvensis, Daucus pusillus, Juniperus virginiana, Lamium amplexicaule, Rhus copallinum, R. glabra, and Torilis arvensis. 


\section{LITERATURE CITED}

Bourlier, B.G., J.D. Nichols, W.J. Ringwold, P.J. Workman, and S. Clemmons. 1979. Osage County Soil Survey. United States Department of Agriculture, Washington, DC.

Branson, C.C., and K.S. Johnson. 1979. Generalized geologic map of Oklahoma. In: Johnson, K.S., C.C. Branson, N.M. Curtis, W.E. Ham, W.E. Harrison, M.V. Marcher, and J.F. Roberts (eds.). Geology and Earth Resources of Oklahoma. Oklahoma Geological Survey, Norman, Oklahoma.

Curtis, N.M. and W.E. Ham. 1979. Geomorphic provinces of Oklahoma. In: Johnson, K.S., C.C. Branson, N.M. Curtis, W.E. Ham, W.E. Harrison, M.V. Marcher, and J.F. Roberts (eds.). Geology and Earth Resources of Oklahoma. Oklahoma Geological Survey, Norman, Oklahoma.

Duck, L.G., and J.B. Fletcher. 1943. A game type map of Oklahoma. In: Duck, L.G., and J.B. Fletcher (eds.). A Survey of the Game and Furbearing Animals of Oklahoma. Oklahoma Department of Wildlife Conservation, Oklahoma City, Oklahoma.

Hoagland, B.W. 2000. The vegetation of Oklahoma: a classification of landscape mapping and conservation planning. Southwest Naturalist 45: 385-420.

Hoagland, B.W. and F.L. Johnson. 2004. Vascular flora of Red Slough and Grassy Slough Wildlife Management Areas, Gulf Coastal Plain, McCurtain County, Oklahoma. Castanea 69: 284296.
Hoagland, B.W. and F.L. Johnson. 2005. Vascular flora of the Deep Fork River in Okmulgee, Creek, and Okfuskee Counties. Publications of the Oklahoma Biological Survey 6: 15-29.

Oklahoma Climatological Survey. 2007. Oklahoma Climatological Data. University of Oklahoma, Norman. (www.ocs.ou.edu accessed 1 August 2007).

Oklahoma Centennial Botanical Garden. 2007. Oklahoma Centennial Botanical Garden research and education center. Tulsa, Oklahoma. www.oklahomacentennialbotanicalgar den.com accessed 1 September 2007).

Oklahoma Natural Heritage Inventory. 2007. Oklahoma Natural Heritage Inventory working list of rare Oklahoma plants. University of Oklahoma, Norman. (www.biosurvey.ou.edu/publicat.html accessed 1 August 2007).

Palmer, M.W., G.L. Wade, and P. Neal. 1995. Standards for the writing of floras. Bioscience 45: 339-345.

Steyermark, J.A. 1963. Flora of Missouri. Iowa State University Press. Ames, Iowa.

Trewartha, G.T. 1968. An Introduction to Climate. McGraw-Hill, New York, New York.

USDA-NRCS. 2007. The PLANTS Database. National Plant Data Center, Baton Rouge, LA 70874-4490 USA. (http://plants.usda.gov accessed 1 May 2007).

Waterfall, U.T. 1973. Keys to the Flora of Oklahoma. Published by the author, Stillwater, Oklahoma. 
Oklahoma Native Plant Record

Volume 7, Number 1, December 2007

Table Summary of floristic collections from the Oklahoma Centennial Botanical Garden site, Osage County, Oklahoma. Format follows Palmer et al. (1995).

\begin{tabular}{lccc}
\hline $\begin{array}{l}\text { Taxonomic } \\
\text { Group }\end{array}$ & Taxa & Native & $\begin{array}{c}\text { Non- } \\
\text { native }\end{array}$ \\
\hline Pteridophyta & 1 & 1 & 0 \\
Coniferophyta & 1 & 1 & 0 \\
Magnoliophyta & 291 & 247 & 44 \\
Magnoliopsida & 199 & 165 & 34 \\
Liliopsida & 92 & 82 & 10 \\
\hline Total & 293 & 249 & 44 \\
\hline
\end{tabular}

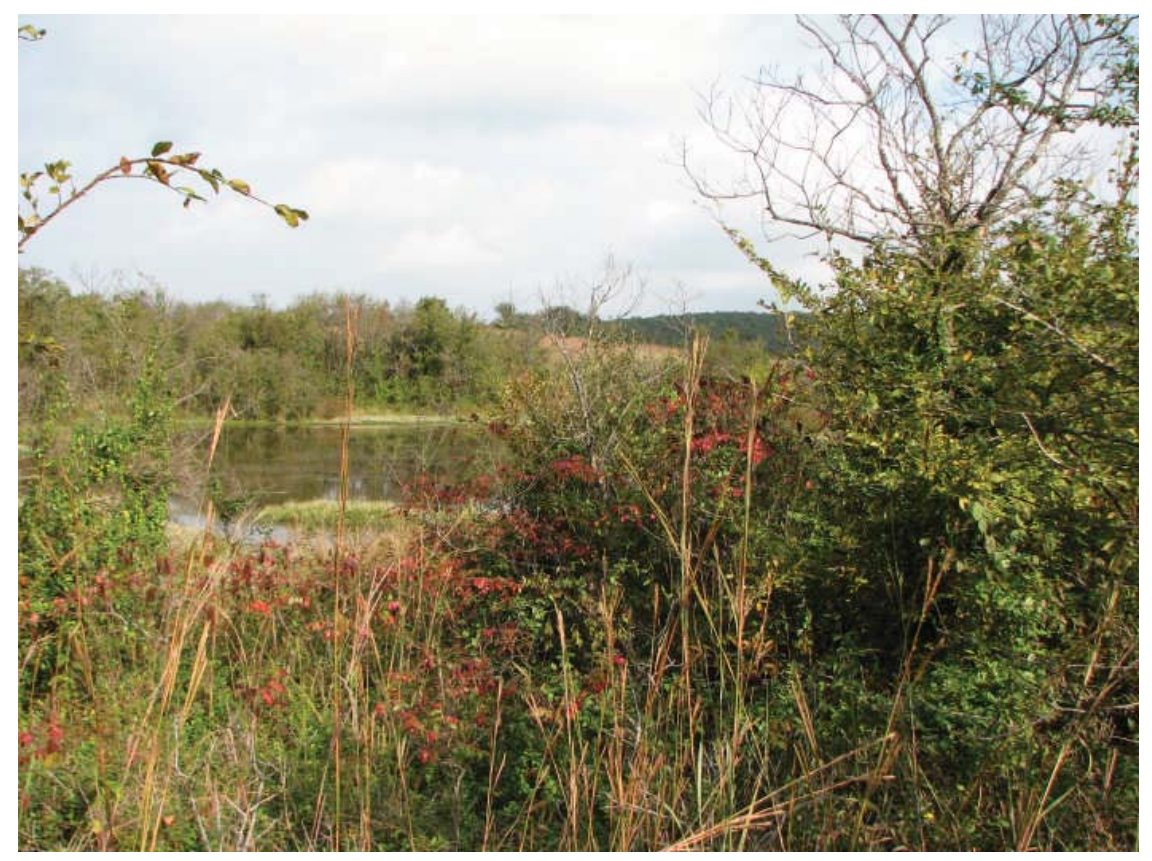

Figure Oklahoma Centennial Botanical Garden site, Osage County, Oklahoma. 


\section{APPENDIX}

Annotated species list for the Oklahoma Centennial Botanical Garden, Osage County, Oklahoma. The first entry is habitat (QSQM=Quercus stellata-Quercus marilandica forest association, SSSN=Schizachyrium scoparium-Sorghastrum nutans grassland association, WETL=wetland and aquatic vegetation, DAOF=disturbed areas and old-field vegetation); followed by the life history ( $A=$ =annual, $B=$ biennial, $P=$ =perennial); habit ( $T=$ tree, $S=$ shrub, $V=$ =woody vine, $H=$ herb, $G=$ graminoid); and collection number. Exotic species are denoted with an asterisk. Voucher specimens were deposited at the Robert Bebb Herbarium of the University of Oklahoma (OKL).

\section{Pteridophyta \\ Aspleniaceae}

Asplenium platyneuron (L.) B.S.P. (ebony

spleenwort) QSQM; P; H; OBG-152

\section{Coniferophyta \\ Cupressaceae}

Juniperus virginiana L. (Eastern red cedar)

QSQM; P; T; OBG-231

\section{Magnoliophyta \\ Magnoliopsida \\ Acanthaceae}

Ruellia humilis Nutt. (fringeleaf wild petunia)

SSSN; P; H; OBG-285

$R$. strepens L. (limestone wild petunia) QSQM;

$\mathrm{P} ; \mathrm{H} ; \mathrm{OBG}-153$

\section{Amaranthaceae}

Amaranthus albus L. (prostrate pigweed)

$$
\text { DAOF; A; H; OBG-012 }
$$

\section{Anacardiaceae}

Rhus aromatica Ait. (fragrant sumac) QSQM;

P; S; OBG-184

R. copallinum L. (flameleaf sumac) SSSN; P; S; OBG-247

R. glabra L. (smooth sumac) SSSN; P; S;

OBG-255

Toxicodendron radicans $\mathrm{L}$. (Kuntze) (poison

ivy) QSQM; P; S; OBG-334

\section{Apiaceae}

Chaerophyllum tainturieri Hook. (hairyfruit chervil) DAOF, QSQM, SSSN; A; H; OBG198
Daucus carota* (Queen Anne's lace) DAOF; B; $\mathrm{H}$; OBG-296

D. pusillus Michx. (American wild carrot)

DAOF, SSSN; A; H; OBG-254

Ptilimnium nuttallii (DC.) Britt. (laceflower)

SSSN; A; H; OBG-304

Spermolepis divaricata (Walt.) Raf. ex Ser. (roughfruit scaleseed) SSSN; A; H; OBG305

Torilis arvensis* (Huds.) Link (spreading hedgeparsley) DAOF; A; H; OBG-256

\section{Aquifoliaceae}

Ilex decidua Walt. ( possumhaw) QSQM; P; S; OBG-144

\section{Asclepiadaceae}

Asclepias viridis Walt. (green antelopehorn) SSSN; P; H; OBG-032

\section{Asteraceae}

Achillea millefolium L. (common yarrow) DAOF, SSSN; P; H; OBG-219

Ambrosia psilostachya DC. (Cuman ragweed) DAOF; P; H; OBG-079

Amphiachyris dracunculoides (DC.) Nutt. (prairie broomweed) DAOF, SSSN; A; H; OBG-097

Antennaria plantaginifolia (L.) Richards. (woman's tobacco) QSQM; P; H; OBG-187

Arnoglossum plantagineum Raf. (groovestem Indian plantain) SSSN; P; H; OBG-221

Carduus nutans ${ }^{*} \mathrm{~L}$. (nodding plumeless thistle) DAOF; B; H; OBG-208

Cirsium altissimum (L.) Hill (tall thistle) SSSN; $\mathrm{P} ; \mathrm{H} ; \mathrm{OBG}-114$ 
Oklahoma Native Plant Record

Volume 7, Number 1, December 2007

C. undulatum (Nutt.) Spreng. (wavyleaf thistle) DAOF, SSSN; P; H; OBG-279

Conyza canadensis (L.) Cronq. (Canadian horseweed) DAOF; A; H; OBG-091

C. ramosissima Cronq. (dwarf horseweed) DAOF; A; H; OBG-089

Coreopsis grandiflora Hogg ex Sweet (largeflower tickseed) SSSN; P; H; OBG300

C. tinctoria Nutt. (golden tickseed) DAOF, SSSN; A; H; OBG-059

Echinacea atrorubens Nutt. (Topeka purple coneflower) SSSN; P; H; OBG-284

Eclipta prostrata (L.) L. (false daisy) WETL; A; $\mathrm{H} ;$ OBG-073

Erigeron strigosus Muhl. ex Willd. (prairie fleabane) DAOF, SSSN; A; H; OBG-015

Eupatorium serotinum Michx. (lateflowering thoroughwort) QSQM; P; H; OBG-062

Euthamia gymnospermoides Greene (Texas goldentop) SSSN; P; H; OBG-148

Gamochaeta purpurea (L.) Cabrera (spoonleaf purple everlasting) -QSQM; P; H; OBG-237

Grindelia lanceolata Nutt. (narrowleaf gumweed) DAOF, SSSN; P; H; OBG048

G. papposa Nesom \& Suh (Spanish gold) DAOF, SSSN; A; H; OBG-020

Helenium amarum (Raf.) H.Rock (yellowdicks) DAOF; A; H; OBG-106

Helianthus hirsutus Raf. (hairy sunflower) QSQM; P; H; OBG-121

Iva angustifolia Nutt. ex DC. (narrowleaf marshelder) WETL; A; H; OBG-125

Krigia caespitosa (Raf.) Chambers (weedy dwarfdandelion) QSQM; A; H; OBG-240

Packera plattensis (Nutt.) W.A. Weber \& A. Löve (prairie groundsel) QSQM; P; H; OBG-192

Pluchea camphorata (L.) DC. (camphor pluchea) WETL; P; H; OBG-011

Pseudognaphalium obtusifolium (L.) Hilliard \& Burtt (rabbit tobacco) DAOF, SSSN; A; H; OBG-002

Pyrrhopappus carolinianus (Walt.) DC. (Carolina desert chicory) DAOF, SSSN; A; $\mathrm{H}$; OBG-280
Rudbeckia hirta L. (blackeyed Susan) SSSN; A; H; OBG-251

Solidago speciosa Nutt. (showy goldenrod) SSSN; P; H; OBG-132

S. ulmifolia Muhl. ex Willd. (elmleaf goldenrod) QMQV; P; H; OBG-049

Sonchus $\operatorname{asper}^{*}$ (L.) Hill (spiny sowthistle) DAOF; $A$; H; OBG-185

Symphyotrichum cordifolium (L.) Nesom (common blue wood aster) QSQM; P; H; OBG-130

S. ericoides (L.) Nesom var. ericoides (white heath aster) DAOF, SSSN; P; H; OBG-154

$S$. lanceolatum (Willd.) Nesom var. lanceolatum (white panicle aster) QSQM, SSSN; P; H; OBG-133

S. oblongifolium (Nutt.) Nesom (aromatic aster) SSSN; P; H; OBG-129

S. patens (Ait.) Nesom (late purple aster) QSQM, SSSN; P; H; OBG-100

S. praealtum (Poir.) Nesom var. praealtum (willowleaf aster) SSSN; P; H; OBG-134

S. subulatum (Michx.) Nesom (Eastern annual saltmarsh aster) DAOF, SSSN, WETL; A; H; OBG-105

Taraxacum officinale* G.H. Weber ex Wiggers (common dandelion) DAOF; P; H; OBG180

Verbesina virginica $\mathrm{L}$. (white crownbeard) QSQM; P; H; OBG-096

Vernonia arkansana DC. (Arkansas ironweed) QSQM; P; H; OBG-007

V. baldwinii Torr. (Baldwin's ironweed) DAOF, SSSN; P; H; OBG-024

Xanthium strumarium $\mathrm{L}$. (rough cocklebur) WETL; A; H; OBG-064

\section{Boraginaceae}

Myosotis verna Nutt. (spring forget-me-not) QSQM; A; H; OBG-267

\section{Brassicaceae}

Brassica nigra* (L.) W.D.J. Koch (black mustard) DAOF; A; H; OBG-195

Capsella bursa-pastoris* (L.) Medik. (shepherd's purse) DAOF; A; H; OBG-327 
Cardamine parviflora L. var. arenicola (Britt.)

O.E. Schulz (sand bittercress) QSQM; A;

H; OBG-194

Erysimum repandum ${ }^{*} \mathrm{~L}$. (spreading wallflower)

DAOF; A; H; OBG-197

Lepidium densiflorum Schrad. (common

pepperweed) DAOF, SSSN; A; H; OBG-

190

\section{Cactaceae}

Opuntia humifusa (Raf.) Raf. (devil's tongue)

QSQM, SSSN; P; S; OBG-169

\section{Callitrichaceae}

Callitriche heterophylla Pursh (twoheaded water-starwort) WETL; A; H; OBG-216

\section{Campanulaceae}

Triodanis biflora (Ruiz \& Pavón) Greene (clasping Venus' looking-glass) SSSN; A; $\mathrm{H}$; OBG-241

Caprifoliaceae

Lonicera japonica* Thunb. (Japanese honeysuckle) QSQM; P; V; OBG-214

Sambucus nigra L. ssp. canadensis (L.) R. Bolli (common elderberry) QSQM; P; S; OBG265

Symphoricarpos orbiculatus Moench (coralberry) QSQM; P; S; OBG-082

Viburnum rufidulum Raf. (rusty blackhaw) QSQM; P; S; OBG-189

\section{Caryophyllaceae}

Arenaria serpyllifolia* $L$. (thymeleaf sandwort)DAOF; A; H; OBG-242

Cerastium pumilum* W. Curtis (European chickweed) DAOF; A; H; OBG-191

Dianthus armeria* L. (Deptford pink) DAOF, SSSN; A; H; OBG-235

Minuartia drummondii (Shinners) McNeill (Drummond's stitchwort) SSSN; A; H; OBG-273

Stellaria media* (L.) Vill. (common chickweed) DAOF; $A ; H ; O B G-176$
Clusiaceae

Hypericum hypericoides (L.) Crantz (St. Andrew's cross) QSQM; P; H; OBG-098

$H$. punctatum Lam. (spotted St. Johnswort) QSQM; P; H; OBG-295

\section{Convolvulaceae}

Convolvulus arvensis* $\mathrm{L}$. (field bindweed) DAOF; P; H; OBG-253

Cornaceae

Cornus drummondii C.A. Mey. (roughleaf dogwood) QSQM; P; T; OBG-232

\section{Cuscutaceae}

Cuscuta cuspidata Engelm. (cusp dodder) DAOF; P; H; OBG-131

\section{Ebenaceae}

Diospyros virginiana L. (common persimmon) QSQM; P; T; OBG-213

\section{Euphorbiaceae}

Acalphya monococca (Engelm. ex Gray) L. Mill. \& Gandhi (slender threeseed mercury) SSSN; A; H; OBG-281

$A$. virginica $L$. (Virginia threeseed mercury) QSQM; A; H; OBG-119

Chamaesyce maculata (L.) Small (spotted sandmat) DAOF; A; $\mathrm{H}$; OBG-023

Croton capitatus Michx. (hogwort) DAOF, SSSN; A; H; OBG-084

C. monanthogynus Michx. (prairie tea) DAOF, SSSN; A; H; OBG-146

C. willdenowii G. L. Webster (Willdenow's croton) SSSN; A; H; OBG-043a

Euphorbia dentata Michx. (toothed spurge) QSQM; A; H; OBG-102

E. heterophylla L. (Mexican fireplant) QSQM; A; $\mathrm{H} ; \mathrm{OBG}-081$

E. spathulata Lam. (warty spurge) SSSN; A; H; OBG-277

Phyllanthus caroliniensis Walt. (Carolina leafflower) DAOF; A; H; OBG-072 


\section{Fabaceae}

Amorpha canescens Pursh (leadplant) SSSN; $\mathrm{P} ; \mathrm{S} ; \mathrm{OBG}-150$

A. fruticosa $L$. (desert false indigo) SSSN, WETL; P; S; OBG-076

Baptisia bracteata Muhl. ex Ell. var. leucophaea (Nutt.) Kartesz \& Gandhi (longbract wild indigo) SSSN; P; H; OBG193

Cercis canadensis L. (Eastern redbud) QSQM; P; T; OBG-208

Chamaecrista nictitans (L.) Moench (partridge pea) DAOF, SSSN; A; H; OBG-087

Crotalaria sagittalis $\mathrm{L}$. (arrowhead rattlebox) SSSN; P; H; OBG-043

Dalea purpurea Vent. (violet prairie clover) SSSN; P; H; OBG-258

Galactia volubilis (L.) Britt. (downy milkpea) QSQM; P; H; OBG-103

Gleditsia triacanthos L. (honeylocust) QSQM; P; T; OBG-115

Kummerowia stipulacea* (Maxin.) Makino (Korean clover) DAOF; A; H; OBG-090

Lathyrus hirsutus* L. (Caley pea) DAOF; A; $\mathrm{H}$; OBG-282

Lespedeza cuneata* (Dum.-cours.) G. Don (Chinese lespedeza) DAOF, SSSN; P; H; OBG-107

L. repens (L.) W. Bart. (creeping lespedeza) QSQM; P; H: OBG-156

L. violacea (L.) Pers. (violet lespedeza) QSQM; $\mathrm{P} ; \mathrm{H} ; \mathrm{OBG}-035$

L. virginica (L.) Britt. (slender lespedeza) QSQM; P; H; OBG-022

Medicago lupulina* L. (black medick) DAOF; A; $\mathrm{H} ;$ OBG-172

Melilotus officinalis* (L.) Lam. (yellow sweetclover) DAOF; A; H; OBG-060

Mimosa nuttallii (DC.) B.L. Turner (Nuttall's sensitive-briar) SSSN; P; H; OBG-264

Pediomelum linearifolium (Torr. \& Gray) J. Grimes (narrowleaf Indian breadfruit) SSSN; P; H; OBG-299

Stylosanthes biflora (L.) B.S.P. (sidebeak pencilflower) SSSN; P; H ; OBG-252

Trifolium dubium ${ }^{*}$ Sibthorp (suckling clover) DAOF; A; H; OBG-243
T. pratense* L. (red clover) DAOF; A; H; OBG290

Vicia villosa* Roth (winter vetch) DAOF; $A$; $H$; OBG-288

\section{Fagaceae}

Quercus muehlenbergii Engelm. (chinkapin oak) QSQM; P; T; OBG-145

Q. shumardii Buckl. (Shumard's oak) QSQM; P; T; OBG-139

Q. stellata Wangenh. (post oak) QSQM; P; T; OBG-083

Q. velutina Lam. (blackjack oak) QSQM; P; T; OBG-335

\section{Gentianaceae}

Sabatia campestris Nutt. (Texas star) SSSN; A; $\mathrm{H} ; \mathrm{OBG}-283$

\section{Geraniaceae}

Geranium carolinianum L. (Carolina geranium) DAOF, SSSN; A; H; OBG-275

\section{Haloragaceae}

Myriophyllum aquaticum* (Vell.) Verdc. (parrot feather watermilfoil) WETL; P; H; OBG-202

\section{Juglandaceae}

Carya illinoinensis (Wangenh.) K. Koch (pecan) QSQM; P; T; OBG-135

C. texana Buckl. (black hickory) QSQM; P; T; OBG-128

\section{Krameriaceae}

Krameria lanceolata Torr. (trailing krameria) SSSN; P; H; OBG-250

\section{Lamiaceae}

Hedeoma drummondii Benth. (Drummond's false pennyroyal) SSSN; P; H; OBG-276

Lamium amplexicaule* $\mathrm{L}$. (henbit deadnettle) DAOF; A; H; OBG-181

L. purpureum* ${ }^{*}$. (purple deadnettle) DAOF; A; $\mathrm{H}$; OBG-182

Monarda fistulosa L. (wild bergamot) QSQM; P; $\mathrm{H} ;$ OBG-260 
Prunella vulgaris L. (common selfheal) QSQM; $\mathrm{P} ; \mathrm{H} ; \mathrm{OBG}-257$

Pycnanthemum tenuifolium Schrad.

(narrowleaf mountain mint) QSQM; P; $\mathrm{H}$; OBG-249

Salvia azurea Michx. ex Lam. (azure blue sage) SSSN; P; H; OBG-093

Scutellaria parvula Michx. (small skullcap)

SSSN; P; H; OBG-302

Teucrium candense $\mathrm{L}$. (Canada germander)

WETL; P; H; OBG-101

Linaceae

Linum medium (Planch.) Britt. (stiff yellow flax)

SSSN; A; H; OBG-031

L. sulcatum Riddell (grooved flax) SSSN; A; H;

OBG-030

\section{Lythraceae}

Ammannia auriculata Willd. (eared redstem)

WETL; A; H; OBG-013

Cuphea viscosissima Jacq. (blue waxweed)

WETL; A; H; OBG-058

\section{Malvaceae}

Callirhoe alcaeoides (Michx.) Gray (light

poppymallow) SSSN; P; H; OBG-270

Menispermaceae

Cocculus carolinus (L.) DC. (Carolina coralbead) QSQM; P; H; OBG-226

\section{Nelumbonaceae}

Nelumbo lutea Willd. (American lotus) WETL;

P; H; OBG-287

\section{Oleaceae}

Fraxinus americana L. (white ash) QSQM; P; T; OBG-155

Ligustrum quihoui ${ }^{*}$ Carr. (waxyleaf privet)

QSQM; P; S; OBG-143

L. sinense* Lour. (Chinese privet) QSQM; P; S; OBG-046

\section{Onagraceae}

Gaura villosa Torr. (wooly beeblossom) SSSN; $\mathrm{P} ; \mathrm{H} ; \mathrm{OBG}-010$

Hoagland \& Buthod
Ludwigia palustris (L.) Ell. (marsh seedbox) WETL; P; H; OBG-001

L. peploides (Kunth) Raven (floating primrosewillow) WETL; P; H; OBG-067

Oenothera linifolia Nutt. (threadleaf eveningprimrose) SSSN; A; H; OBG-220

\section{Oxalidaceae}

Oxalis stricta L. (common yellow oxalis) DAOF, SSSN; P; H; OBG-095

O. violacea (violet woodsorrel) QSQM, SSSN; $\mathrm{P} ; \mathrm{H} ; \mathrm{OBG}-199$

\section{Passifloraceae}

Passiflora incarnata L. (purple passionflower) SSSN; P; H; OBG-248

\section{Plantaginaceae}

Plantago aristata Michx. (largebraced plantain) QSQM; A; H; OBG-225

$P$. virginica L. (Virginia plantain) SSSN; $A ; H$; OBG-271

\section{Polygalaceae}

Polygala incarnata $\mathrm{L}$. (procession flower)

SSSN; A; H; OBG-274

\section{Polygonaceae}

Polygonum pensylvanicum L. (Pennsylvania smartweed) WETL; A; H; OBG-298

P. punctatum Ell. (dotted smartweed) WETL; $A$; $\mathrm{H} ;$ OBG-071

Rumex crispus* L. (curly dock) DAOF, WETL; $\mathrm{P} ; \mathrm{H} ; \mathrm{OBG}-209$

\section{Portulacaceae}

Phemeranthus parviflorum (Nutt.) Kiger

(sunbright) SSSN; P; H; OBG-033

Portulaca oleracea* L. (little hogweed) DAOF; A; H; OBG-108

\section{Primulaceae}

Samolus ebracteatus Kunth (limewater brookweed) WETL; P; H; OBG-137 
Oklahoma Native Plant Record

Volume 7, Number 1, December 2007

\section{Ranunculaceae}

Delphinium carolinianum Walt. (Carolina larkspur) SSSN; P; H; OBG-229

\section{Rosaceae}

Amelanchier arborea (Michx. f.) Fern. (common serviceberry) QSQM; P; T; OBG-183

Crataegus mollis Scheele (Arnold hawthorn) QSQM; P; S; OBG-167

C. viridis L. (green hawthorn) QSQM; P; S; OBG-186

Prunus mexicana S. Wats. (Mexican plum) QSQM; P; T; OBG-110

P. serotina Ehrh. (black cherry) QSQM; P; T; OBG-045

Rosa multiflora* Thunb. ex Murr. (multiflora rose) QSQM; P; V; OBG-263

$R$. setigera Michx. (climbing rose) SSSN; P; V; OBG-292

Rubus sp. (blackberry) DAOF, QSQM; P; V; OBG-204

\section{Rubiaceae}

Cephalanthus occidentalis L. (common buttonbush) WETL; P; S; OBG-050

Diodia teres Walt. (poorjoe) SSSN; A; H; OBG070

Galium aparine L. (stickywilly) DAOF, QSQM; A; H; OBG-233

G. pilosum Ait. var. puncticulosum (Michx.) Torr. \& Gray (hairy bedstraw) QSQM; P; H; OBG-061

G. virgatum Nutt. (southwestern bedstraw) SSSN; A; H; OBG-233

Houstonia pusilla Schoepf (tiny bluet) SSSN; A; $\mathrm{H}$; OBG-174

Sherardia arvensis ${ }^{*} \mathrm{~L}$. (blue fieldmadder) DAOF; A; H; OBG-177

\section{Salicaceae}

Populus deltoides Bartr. ex Marsh. (Eastern cottonwood) WETL; P; T; OBG-118

Salix nigra Marsh. (black willow) WETL; P; T; OBG-051

\section{Sapotaceae}

Sideroxylon lanuginosum Michx. (gum bully) QSQM; P; T; OBG-052

\section{Scrophulariaceae}

Bacopa rotundifolia (Michx.) Wettst. (disk waterhyssop) WETL; P; H; OBG-109

Castilleja indivisa Engelm. (entireleaf Indian paintbrush) SSSN; A; H; OBG-200

Lindernia dubia (L.) Pennell (yellowseed false pimpernel) WETL; A; H; OBG-078

Nuttallanthus canadensis (L.) D.A. Sutton (Canada toadflax) SSSN; A; H; OBG-272

Veronica polita* Fries (gray field speedwell) DAOF; A; H; OBG-173

\section{Solanaceae}

Physalis pubescens L. (husk tomato) SSSN; A; $\mathrm{H} ;$ OBG-014

Solanum americanum $P$. Mill. (American black nightshade) QSQM; A; H; OBG-017

$S$. carolinense L. (Carolina horsenettle) DAOF, SSSN; P; H; OBG-075

$S$. elaeagnifolium Cav. (silverleaf nightshade) DAOF, SSSN; P; H; OBG-116

$S$. rostratum Dunal (buffalobur nightshade) DAOF; A; H; OBG-075

\section{Ulmaceae}

Ulmus alata Michx. (winged elm) QSQM; P; T; OBG-245

U. rubra Muhl. (slippery elm) QSQM; P; T; OBG-246

\section{Valerianaceae}

Valerianella radiata (L.) Dufr. (beaked cornsalad) SSSN; A; H; OBG-268

\section{Verbenaceae}

Verbena bracteata Cav. ex Lag. \& Rodr. (bigbract verbena) DAOF, SSSN; A; H; OBG-113

V. stricta Vent. (hoary verbena) DAOF; $\mathrm{P} ; \mathrm{H}$; OBG-278

V. urticifolia L. (white vervain) QSQM; P; H; OBG-141 


\section{Vitaceae}

Parthenocissus quinquefolia (L.) Planch.

(Virginia creeper) QSQM; P; V; OBG-222

Vitis cinerea (Engelm.) Millard (graybark grape)

QSQM; P; V; OBG-297

\section{Zygophyllaceae}

Tribulus terrestris* ${ }^{*}$ L. (puncturevine) DAOF; A; H; OBG-019

\section{Liliopsida \\ Alismataceae}

Alisma subcordatum Raf. (American water plantain) WETL; P; H; OBG-286

Sagittaria ambigua J.G. Sm. (Kansas arrowhead) -WETL ; P; H; OBG-266

$S$. latifolia Willd. (broadleaf arrowhead) -WETL ; P; H; OBG-027

\section{Commelinaceae}

Commelina erecta L. (whitemouth dayflower) DAOF, SSSN; P; H; OBG-028

Tradescantia ohiensis Raf. (bluejacket) SSSN; $\mathrm{P} ; \mathrm{H} ; \mathrm{OBG}-236$

\section{Cyperaceae}

Carex aggregata Mackenzie (glomerate sedge) QSQM; P; G; OBG-328

C. blanda Dewey (Eastern woodland sedge) QSQM; P; G; OBG-326

C. festucacea Schkuhr ex Willd. (fescue sedge) QSQM; P; G; OBG-330

C. frankii Kunth (Frank's sedge) -WETL; P; G; OBG-163

C. microdonta Torr. \& Hook. (littletooth sedge) SSSN; P; G; OBG-203

Cyperus croceus Vahl (Baldwin's flatsedge) SSSN; P; G; OBG-166

C. echinatus (L.) Wood (globe flatsedge) SSSN; P; G; OBG-099

C. odoratus L. (fragrant flatsedge) WETL; $A ; G$; OBG-164

C. squarrosus L. (bearded flatsedge) DAOF; A; G; OBG-065

C. strigosus L. (strawcolored flatsedge) SSSN; P; G; OBG-161
C. virens Michx. (green flatsedge) WETL; P; G; OBG-147

Eleocharis lanceolata Fern. (daggerleaf spikerush) WETL; A; G; OBG-325

E. obtusa (Willd.) J.A. Schultes (blunt spikerush) WETL; A; G; OBG-127

E. palustris (L.) Roemer \& J.A. Schultes (common spikerush) WETL; P; G; OBG324

Fimbristylis autumnalis (L.) Roemer \& J.A. Schultes (slender fimbry) WETL; A; G; OBG-069

F. puberula (Michx.) Vahl (hairy fimbry) WETL; P; G; OBG-322

F. vahlii (Lam.) Link (Vahl's fimbry) WETL; A; G; OBG-063

Isolepis carinata Hook. \& Arn. ex. Torr. (keeled bulrush) WETL; A; G; OBG-239

Rhynchospora globularis (Chapman) Small (globe beaksedge) -WETL; P; G; OBG-323

Scirpus pendulus Muhl. (rufous bulrush) WETL; P; G; OBG-244

\section{Iridaceae}

Sisyrinchium campestre Bickn. (prairie blueeyed grass) SSSN; P; H; OBG-201

Juncaceae

Juncus brachycarpus Engelm. (whiteroot rush)

WETL; P; G; OBG-318

J. diffusissimus Buckl. (slimpod rush) WETL; P; G; OBG-055

J. interior Wieg. (inland rush) SSSN; P; G; OBG-321

J. marginatus Rostk. (grassleaf rush) WETL; P; G; OBG-319

J. tenuis Willd. (poverty rush) WETL; P; G; OBG-168

\section{Lemnaceae}

Lemna minor L. (common duckweed) WETL; $\mathrm{P} ; \mathrm{H} ; \mathrm{OBG}-332$

Wolffia columbiana (Columbian watermeal) WETL; P; H; OBG-333 
Oklahoma Native Plant Record

Volume 7, Number 1, December 2007

\section{Liliaceae}

Allium canadense (meadow garlic) SSSN; P; $\mathrm{H}$; OBG-188

Erythronium mesochoreum Knerr (midland fawnlily) QSQM; P; H; OBG-301

Hypoxis hirsuta (L.) Coville (common goldstar) SSSN; P; H; OBG-217

Nothoscordum bivalve (L.) Britt. (crowpoison) SSSN; P; H; OBG-179

\section{Orchidaceae}

Spiranthes vernalis Engelm. \& Gray (spring ladies'-tresses) SSSN; P; H: OBG-291

\section{Poaceae}

Aegilops cylindrica* Host (jointed goatgrass) DAOF; A; G; OBG-210

Agrostis hyemalis (Walt.) B.S.P. (winter bentgrass) SSSN; P; G; OBG-307

A. perennans (Walt.) Tuckerman (upland bentgrass) QSQM; P; G; OBG-306

Andropogon gerardii Vitman (big bluestem) SSSN; P; G; OBG-112

A. virginicus L. (Virginia wildrye) SSSN; P; G; OBG-117

Aristida dichotoma Michx. (churchmouse threeawn) SSSN; A; G; OBG-038

Bothriochloa laguroides (DC.) Herter (silver beardgrass) DAOF, SSSN; P; G; OBG-005

Bromus catharticus* Vahl (rescuegrass) DAOF; A; G; OBG-206

B. tectorum ${ }^{*}$ L. (cheatgrass) DAOF; A; G; OBG-309

Buchloe dactyloides (buffalograss) SSSN; P; G; OBG-218

Danthonia spicata (L.) Beauv. ex Roemer \& J.A. Schultes (poverty oatgrass) QSQM; P; G; OBG-207

Dichanthelium acuminatum (Sw.) Gould \& C.A. Clark var. fasciculatum (Torr.) Freckmann (Western panicgrass) QSQM; P; G; OBG124

D. depauperatum (Muhl.) Gould (starved panicgrass) QSQM; P; G; OBG-311

D. malacophyllum (Nash) Gould (softleaf rosette grass) QSQM; P; G; OBG-120
D. scoparium (Lam.) Gould (velvet panicum) QSQM; P; G; OBG-317

D. villosissimum (Nash) Freckmann (whitehair rosette grass) QSQM; P; G; OBG-310

Digitaria cognata (J.A. Schultes) Pilger (Carolina crabgrass) DAOF; P; G; OBG003

D. sanguinalis (L.) Scop. (hairy crabgrass) DAOF; A; G; OBG-004

Echinochola crus-galli* (L.) Beauv. (barnyardgrass) WETL; A; G; OBG-025

Eleusine indica* ${ }^{*}$ (L.) Gaertn. (Indian goosegrass) DAOF; A; G; OBG-068

Elymus virginicus L. (Virginia wildrye) QSQM, SSSN; P; G; OBG-312

Eragrostis barrelieri* Daveau (Mediterranean lovegrass) DAOF; A; G; OBG-008

E. intermedia A.S. Hitchc. (plains lovegrass) QSQM; P; G; OBG-021

E. spectabilis (Pursh) Steud. (purple lovegrass) DAOF, SSSN; P; G; OBG-018

Hordeum pusillum Nutt. (little barley) SSSN; A; G; OBG-211

Leersia oryzoides (L.) Sw. (rice cutgrass) WETL; P; G; OBG-314

L. virginica Willd. (whitegrass) -WETL; P; G; OBG-140

Lolium perenne* L. (perennial ryegrass) DAOF; P; G; OBG-230

Muhlenbergia sobolifera (Muhl. ex Willd.) Trin. (rock muhly) QSQM; P; G; OBG-029

Panicum anceps Michx. (beaked panicgrass) QSQM, SSSN; P; G; OBG-104

$P$. dichotomiflorum Michx. (fall panicgrass) WETL; A; G; OBG-122

P. philadelphicum Bernh. ex Trin. (Philadelphia panicgrass) SSSN; A; G; OBG-123

$P$. rigidulum Bosc ex Nees (redtop panicgrass) WETL; P; G; OBG-162

$P$. virgatum L. (switchgrass) SSSN; P; G; OBG094

Paspalum pubiflorum Rupr. ex Fourn. (hairyseed paspalum) SSSN, WETL; P; G; OBG-053

Phalaris caroliniana Walt. (Carolina canarygrass) WETL; A; G; OBG-228 
Poa annua* L. (annual bluegrass) QSQM; A; G; OBG-178

Schedonnardus paniculatus (Nutt.) Trel. (tumblegrass) DAOF, SSSN; P; G; OBG057

Schedonorus phoenix (Scop.) Holub DAOF; P; G; OBG-238

Schizachyrium scoparium (Michx.) Nash (little bluestem) SSSN; P; G; OBG-149

Setaria parviflora (Poir.) Kerguélen (marsh bristlegrass) WETL; P; G; OBG-158

S. pumila* (Poir.) Roemer \& J.A. Schultes (yellow foxtail) DAOF; A; G; OBG-009

Sorghastrum nutans (L.) Nash (Indian grass) SSSN; P; G; OBG-336

Sorghum halepense* (L.) Pers. (Johnsongrass) DAOF, SSSN; P; G; OBG-111

Sphenopholis obtusata (Michx.) Scribn. (prairie wedgescale) QSQM; P; G; OBG-308

Sporobolus cryptandrus (Torr.) Gray (sand dropseed) SSSN; P; G; OBG-016
Steinchisma hians (Ell.) Nash (gaping grass) WETL; P; G; OBG-262

Tridens strictus (Nutt.) Nash (longspike tridens) DAOF, SSSN; P; G; OBG-044

Vulpia elliotea (Raf.) Fern. (squirreltail fescue) SSSN; A; G; OBG-315

V. octoflora (Walt.) Rydb. (sixweeks fescue) SSSN; A; G; OBG-315

\section{Potamogetonaceae}

Potamogeton diversifolius Raf. (waterthread pondweed) WETL; P; H; OBG-160

\section{Smilacaceae}

Smilax rotundifolia $\mathrm{L}$. (roundleaf greenbriar) QSQM; P; V; OBG-223

$S$. tamnoides L. (bristly greenbriar) QSQM; P; V; OBG-224

\section{Typhaceae}

Typha domingensis Pers. (southern cattail) WETL; P; H; OBG-126 